\title{
A Study on Extraction and Comparison of Digital Content Key Frame in UCC Service Environment
}

\author{
Eun-Gyeom Jang ${ }^{\dagger}$
}

\begin{abstract}
In this paper, we proposed a mechanism that prevents indiscreet use of digital contents, verifies created content's copyrights to provide services to granted user and protects digital contents by law by authenticating the original digital content whenever an infringement of copyright occurs in UCC environments. The proposed mechanism uses specific information and features of contents as copyrights authentication information without additional information. Also, provides the fact of violation by inferring the modification of the original digital contents. That means this mechanism infers same or similar value from the contents; fraudulent use of content, modification of content color, modification of content format, modification of content resolution and illegal use of frame not principal key frame. In that point, we found that the inferred value from the content differed according to features of content modification.
\end{abstract}

Key words: Copyright Protection, Contents Authentication, User Created Contents, Original Authentication.

\section{INTRODUCTION}

Web 2.0 presented in early 2000 is out of existing HTML system and has called for the participation of users. The most representative feature in Web 2.0 is UCC. UCC involves not only moving images but also everything expressed by a digital type such as text content and music content. UCC has been widely used in people's lives like a cellular phone or PDA not limited in just only computers. However, UCC files circulating in the world wide web are illegal copies not pure creations. Moving image UCC files have more copies than other content does because the creators who can make moving image content by themselves are very limited. Accordingly, ones have used the ways of copying some part of popular content which had been al-

* Corresponding Author: Eun-Gyeom Jang, Address: (300-716) 96-3 Yongun-dong, Dong-gu, Daejeon, Korea, TEL : +82-42-280-2541, FAX : +82-42-280-2889, E-mail: jangegu@dju.kr

Receipt date: Jan. 12, 2010, Revision date: July 1, 2011 Approval date: July 18, 2011

Dept. of Computer Engineering, Daejeon University ready made or adding the editing in normal cases. In addition, the illegal copying of content which is popular with young generations has been showed more frequently in programs and animations provided by broadcasting networks[1].

Pure created UCC files does not cause copyright piracy but illegal copies could bring about piracy problems for originals. In the same way, UCC made by compositing or combining several other UCC also could cause piracy of each original UCC itself. These pure creative UCC files and revised ones would have problems caused by involving distorted images and information[2,3].

In this paper, we proposed The original authentication mechanism of UCC to protect copyright from the cases of libel, malicious code, and copy ${ }^{-}$ right piracy caused by unadvisedly making UCC.

\section{IMAGE FRAME DETECTION AND COMPARISON MECHNISM}

\subsection{Pixel-based Comparison method and His- tory Comparison method}

The Pixel-based Comparison method uses the 
feature that there are low changes of pixel rate in the same shot. $F_{i}(x, y)$ means pixel rate of $(x, y)$ in the $i$ frame. The corresponding difference of adjacent frame becomes 1 if the difference of pixel value exceeds the critical value t. (See Formula 2.1)

$$
D P_{i}(x, y)= \begin{cases}1 & \text { if }\left|F_{i}(x, y)-F_{i+1}(x, y)\right|>t \\ 0 & \text { otheruise }\end{cases}
$$

Formula 2.2 means that the pixel rate is considered as a cut in case of it exceeds the particular critical value $T_{p} . \mathrm{X}$ and $\mathrm{Y}$ in formula 2.2 mean the height and width of the image.

$$
\frac{\sum_{x, y=1}^{X, Y} D P_{i}(x, y)}{X * Y} * 100>T_{p}
$$

This pixel-based comparison mechanism detects the wrong cutback through the movement of camera and object. It is the camera movements like fade and zoom in/out and object movements to make many pixels change. As a result, the changes can show the wrong cutback[2,3].

However, history comparison method is common method using the feature that color distribution is similar in frames of the same shot. Cutback is detected by comparing the difference between histograms in the adjacent frame of moving image with critical value $T_{h}$. (See Formula 2.3)

$i$ means the number of frame and $j$ means the color value of histogram. $H_{i}(j)$ presents the frequency of given color value.

$$
D_{i}=\sum_{j=1}^{N}\left|H_{i}(j)-H_{i+1}(j)\right| T_{h}
$$

This histogram comparison method is more faithful than pixel-based comparison method because histogram comparison method can detect accurate cutback in movements as the total histogram hardly change in spite of fast movement of the object. However, histogram comparison method could detect wrong cutback in the brightness change. In other words, it could detect wrong cutback in case of the abrupt lightening changes, flash, and similar background or $\operatorname{mood}[4-6]$.

\subsection{The Comparison method through Entropy}

Formula 2.4 expresses the complexity of image data based on probability. We can easily calculate the image complexity by using this entropy.

$$
E=-\sum_{j=0}^{K} P\left(a_{j}\right) \log P\left(a_{j}\right)
$$

$a_{j}$ is the number of the $j$ th pixel value and $P\left(a_{j}\right)$ is probability of $a_{j}$.

The difference of frames can be detected by measuring the difference of entropy between successive two frames in a moving image. When happening abrupt lightening changes, the distribution of color histogram changes differently. However, entropy does not change much because the $\mathrm{com}^{-}$ plexity of the image hardly changes although there are some lightness changes of objects, background, or total image. The wrong cutback caused by lightening changes can be prevented by using the difference of entropy between two successive frames. Therefore, a cutback is accepted if $D_{\text {total }}$ is over critical value after calculating the difference of color histogram and entropy between two frames through color histogram and entropy comparison method[4,6].

In formula 2.5 presents the entropy difference of two frames and $D_{h}$ means the difference of color histogram (See Formula 2.6), $D_{e}$ is the total difference. The difference of two frames can be shown as the sum of the difference of color histogram and the difference of entropy.

$$
\begin{aligned}
& D_{e}=E_{m}-E_{m+1} \\
& D_{\text {total }}=D_{b}+w D_{e} \\
& D_{\text {total }}>T_{e}
\end{aligned}
$$

$w$ is weighted value and the cutback was regarded when the difference of two frames is over critical value as shown in Formula 2.7. The wrong cutback is not detected in abrupt lightening changes on entropy comparison method but is de- 
tected when lightness changes gradually like fade.

\subsection{The Comparison method through Edge Image in Compression Area}

Low frequency edge intensity, $P_{L}$ is calculated using five coefficients among low frequency of $\mathrm{AC}$ coefficient shown in Formula 2.8. Edge image is calculated by getting edge block comparing $P_{L}$ with the critical value. Scene-change detection is conducted by comparing equatorial edge histogram $P_{H}^{\prime}$ and axial edge histogram $P_{v}^{\prime}$ of the reference frame compensated movements with $P_{H}$ and $P_{V}$ of current frame shown in Formula 2.9.

$$
\begin{aligned}
& P_{L}=F_{01}^{2}+F_{10}^{2}+F_{02}^{2}+F_{20}^{2}+F_{11}^{2} \\
& \text { where, } F_{i, j}:(i, j), \text { DCT coefficient } \\
& d(k, k+1)=\sum_{x=0}^{M-1} \mid P_{H}(x+u) \\
& -P_{H}(x)\left|+\sum_{y=0}^{N-1}\right| P_{V}(y+v)-P_{v}(y) \mid
\end{aligned}
$$

where, $M, N$ : width and length of edge image $u, v$ : Vertical and Horizontal of moving vecter

It is the most attractive feature that the result of scene-change detection is accurate when edge image is acquire better by using DCT coefficient than by using $\mathrm{AC}$ coefficient[7-9].

Edge image is acquired after dct_dc_size decodes using the feature that the code length of DC coefficient(dct_dc_size involved MPEG syntax) become elongated in the block which have flexible lightness value. There is one advantage that the decoding process to get data used in algorithm is shortened.

\subsection{Pros and Cons of The Conventional Mechanisms}

The problem about copyright of content creator is on top of all content including UCC. Piracy is judged at court by comparing original content with infringing content in offline. It is easy at court to prove the piracy comparing original content with infringing content. The question is raised; "How can the piracy be proved by the changed of original content?" Of course, watermarking is provided to protect copyright currently. Watermarking is useful if some or all of the original content is copied when not being changed. However, the content having damaged watermark or being changed does not guaranteed protection of copyright.

This paper uses the key frame in order to prevent contents from copy, color change, frame copy, and brightness change of digital content. Copy can be easily noticed by using the key frame because digital content forms frames based on the key frame.

\section{DIGITAL CONTENT VIDEO COM- PARISON MECHANISM}

\subsection{Video Key Frame Extraction Algorithm}

Original content is compared with target content with the key frame extracted a moving image shown as Figure 1.

- Sampling: A process of changing RGB color system to $\mathrm{Y}-\mathrm{Cb}-\mathrm{Cr}$ color system

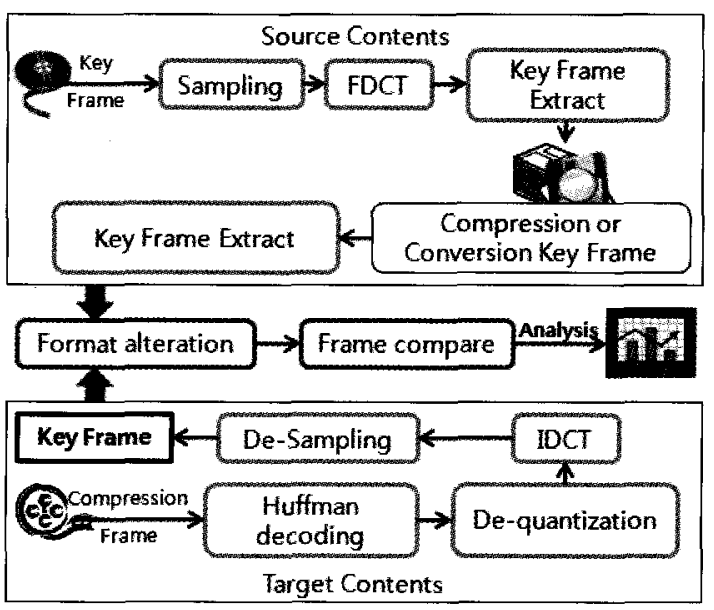

Fig. 1. Extraction and Comparison algorithm of Digital Content's Key Frame. 
- DCT: A process of changing sampling data to the sum of cosine function

- Quantization: A process of eliminating unnecessary constituent

- Huffman coding: Data compression

Compression is conducted by quantization and Huffman coding. Huffman coding is a lossless compression algorithm but quantization is different from original image after image restoration. However, in case of a moving image, some loss does not have an influence on image quality. In addition, the reverse of compression method is the way of image restoration. Key frame make color system modified by sampling. This modified date is modified as cosine function into quantization process. A result from DCT process is compressed and managed with Huffman coding because data could be lost in quantization process. Also, this information is used as authentication of digital content.

\subsection{Digital Content Video Comparison $\mathrm{Me}^{-}$ chanism}

Extracted key frame is the form of compressed video. DC image extraction method is used to compare original key frame with target key frame. Similarity between representative frames is discriminated as extracting DC image

FFD(Frame-to-Frame Difference) which indicates change extent is calculated by using the difference between pixel value and histogram of extracted DC image. Extracted DC images would be different frames if the difference between sec- $^{-}$ ond maximum values in windows is over critical value with moving sliding window upward FFD value. DC image needs much fewer calculations than decoding total image to get original image. Figure 2 is video comparison algorithm.

Key frame discrimination algorithm applies the first discrimination algorithm using luminance and $\mathrm{DC}$ value of chroma block. The first discrimination

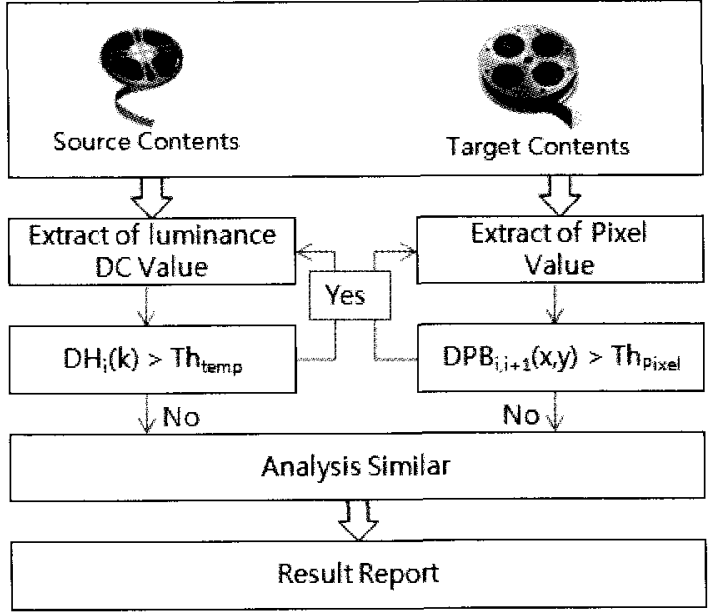

Fig. 2. Video Comparison Algorithm.

algorithm conducts to extract luminance value of frame and DC value of chroma block. These values of original key frame and target key frame compare with certain critical value.

If the compared value is smaller that critical value, the second discrimination algorithm is applied. The second discrimination algorithm could interpret that the frames are similar when the value to get compare original key frame with target key frame using pixel value is smaller than critical value. In other word, if the value that is taken in the second discrimination algorithm is bigger than critical value, the target frame is regarded as a illegally copied frame.

\subsection{Comparison With Frame Luminance And DC Value Of Chroma Value}

The first similar frame discrimination method is using the similarity discrimination algorithm using luminance and $\mathrm{DC}$ value between frames shown in Formula 3.1.

$D H_{i, j}(k)=\sum_{k=0}^{K-1}\left|H_{i}(k)-T_{j}(k)\right|>T h_{t e m p}$

$K$ : total amount of brightness or color level

$H_{i}(k)$ : histogram function which has brightness value $k$ in $i^{\text {th }}$ frame

$\left|H_{i}(k)-T_{j}(k)\right|$ : difference of the absolute value of 
histogram which has brightness $k$ between the original $i$ frame and targeted $j$ frame

$D H_{i, j}(k)$ : sum of the absolute value of the difference of histogram where it shows the similarity between the original $i$ frame and the targeted $j$ frame

$T h_{\text {temp }}:$ similarity critical value

The frames are similar if $D H_{i, j}(k)$ value is smaller than similarity critical value and vice versa. $T h_{\text {temp }}$ could judge the similarity extent.

The second similar frame discrimination algorithm is applied to $D H_{i, j}(k)$ when it is smaller than critical value. The second discrimination algorithm compensate the first one because the first one is inefficiently affected by the brightness of color tone. Also, if $D H_{i, j}(k)$ is bigger than critical value, these frames are not similar. And then the first algorithm can read the next frame and apply the first similar frame discrimination method.

\subsection{Frame Pixel Value Comparison}

The second similarity frame discrimination algorithm is the result of the first one and is applied when $D H_{i, j}(k)$ is smaller than critical value. In addition, the second algorithm is using the pixel.

If the sum of absolute value of the pixel value's difference between two frames being the same position is over critical value, there is not similarity between two frames. Similarity discrimination using the pixel value's difference is taken by Formula 3.2 .

$$
D P B_{i, j}(x, y)=\sum_{x=0}^{M-1} \sum_{y=0}^{N-1} \Delta P_{i, j}(x, y)>T h_{p i x e l}
$$

$M$ : vertical pixel value

$N$ : horizontal pixel value

$P_{i}(x, y)$ : brightness value of pixel at point $(x, y)$ in $i$ frame

$\Delta P_{i, j}(x, y)$ : difference of the absolute value of pixel brightness at point $(x, y)$ in $i$ frame and $j$ frame $\left(\left|P_{i}(x, y)-P_{j}(x, y)\right|\right)$
$D P B_{i, j}(x, y)$ : sum of the absolute value of difference in brightness which shows the similarity between $i$ frame and $j$ frame $T h_{\pi x e l}$ : similarity critical value

There is very small difference value of pixels in similar frames, and vice versa. Similarity of pixels shows similarity between frames using certain critical value. If $D P B_{i, j}(x, y)$ is over $T h_{\text {pixel }}$, these frames are not similar. On the other hand, if $\eta_{h_{\text {pixel }}}$ is under critical value, similar frame detection values must be analyzed to determine similarity.

Without question, the first or second similar frame discrimination algorithm depends on critical value. In case critical value sets up smaller, the result is more accurate but it is difficult to find out content piracy. Also, in case critical value sets up bigger, the content not conducting piracy is elected as a result. The best critical value is taken by controlling critical value in the performance test of algorithm.

\section{TEST AND ANALYSIS OF THE PRO- POSED MECHANISM}

To test performance of the proposed mechanism, the target content of copyright piracy is tested by applying forms of 'original content', 'the content modified resolution', 'the content modified format', and 'the content modified representative key frame'.

Similarity applied to $D H$ and $D P B$ is judged by comparing with critical value. 0.1 as the standard for judgement of critical value is applied to $D H$ and $D P B$. It shows almost 90 percent of similarity when critical value is 0.1 .

\subsection{Digital Content Experiment by Type}

There are four cases of piracy or modification of original content in this chapter.

(1) Using The Original Frame Without Modification

Using original frame without modification means 
the case of copying total original content or some of it. Using is clearly turned out because the same representative key frame is used. Figure 3 is the result of $D H$ and $D P B$.

Figure 3 shows the result of comparing the representative key frame without modification. $D H$ and $D P B$ of every original frame has about $100 \%$ similarity value. But, as an experimental result, we earned the $98.8 \%$ result at the fifth frame. The fifth frame show the result is 0.022 to insert the noise.

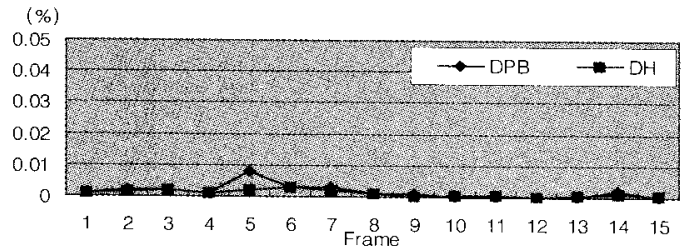

Fig. 3. Comparison of DH/DPB Frame.

\section{(2) Chroma Block Modification}

Chroma block modification means the case of modifying the brightness like making a frame be gray. In this case, brightness is modified but the basic structure and format is maintained. Figure 4 shows $D H$ and $D P B$ value applied to the modified frame in chroma block.

When frame color is modified, the average $D H$ value is over $98.5 \%$ and the average $D P B$ value is over $94.2 \%$. It means that $D P B$ is sensitive to the change of brightness and $D H$ is effective to discriminate similarity.

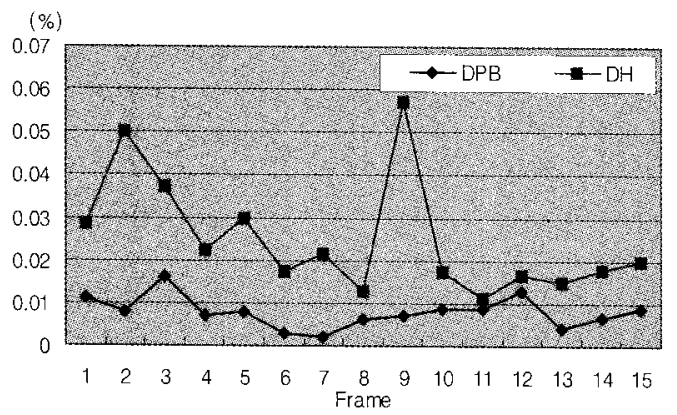

Fig. 4. DH/DPB comparison that followed chroma block's modification.

\section{(3) File Format Modification}

Resolution could change when original frame's format is modified to another file's format. Figure 5 shows comparison value of $D H$ and $D P B$ that followed file format's modification.

The modification of resolution and file format shows the difference in the method or format of compression. The result that $D P B$ is over $D H$ means the modified frame of file format or resolution is better than $\mathrm{DH}$.

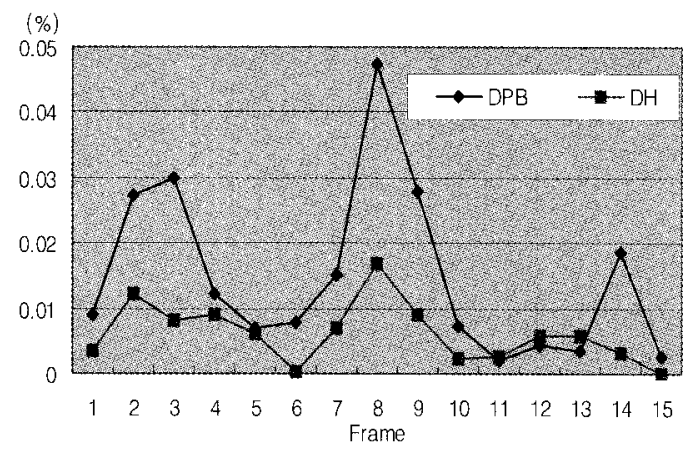

Fig. 5. DH/DPB comparison that followed file format's modification

(4) Illegal Use of a Certain Frame

This case includes to produce the second content using the essential image of original image illegally. Figure 6 shows the result of comparison.

$D H$ is over $88.6 \%$ and $D P B$ is over $86.5 \%$ as a comparison value.

$D H$ is more efficient than $D P B$ but there is not much difference between them.

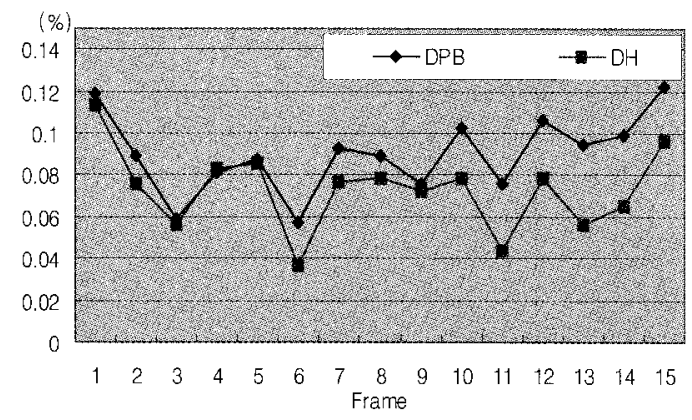

Fig. 6. Illegal Use of Middle Frame 
$D H$ and $D P B$ is almost similar in similarity.

(5) Result Analysis of algorithm Application based on the Content Feature

Table 1 summarizes the result of algorithm application on (1) to (4) related to the modification or revision of original content.

Both $D H$ and $D P B$ are efficient in 'the frame without modification of original frame'. $D H$ is more efficient than $D P B$ in 'chroma block modification'. $D H$ is more efficient than $D P B$ in 'file format modification' and 'illegal use of middle frame'.

This paper properly utilizes the features of two algorithms. In addition, $D H$ is not inefficient because it is based on not the probability of similarity but the accuracy of similarity to prove copyright piracy.

\subsection{Result Analysis and Evaluation of Pro- posed Mechanism}

The modified content in the original or the for- mat is discriminated by setting a critical value to the digital content by type as ' 0.1 '. High accuracy is presented when critical value is set to 0.1 .

Comparison value to identify similarity has a different set point based on the content format. It is efficient to apply the modified set point when the content form is recognized in advance. However, because it is difficult to know the content format, comparison value is calculated after recognizing the content format using 'the original content without modification'. Also, 'Equivalent' menas that two content are similar in the 95 percent when comparison value is 0.000 to 0.049 , 'Similar' means that they are similar in the 75 percent and over, and 'Different' means that they are in the 76 percent and less.

Performance of this proposed method is tested by comparing with extraction method for screen conversion in moving image shown in Table 3.

Table 1. Result Analysis of algorithm Allocation based on the Content Feature

\begin{tabular}{|c|c|c|c|}
\hline Frame Feature & $\begin{array}{l}\text { DH comparison } \\
\text { value }\end{array}$ & $\begin{array}{l}\text { DPB comparison } \\
\text { value }\end{array}$ & Result Analysis \\
\hline $\begin{array}{l}\text { The frame without modification } \\
\text { of original frame }\end{array}$ & over 0.991 & over 0.988 & Both DH and DPB are efficient. \\
\hline Chroma block modification & over 0.943 & over 0.986 & DPB is more efficient than $\mathrm{DH}$. \\
\hline File format modification & over 0.981 & over 0.951 & $\mathrm{DH}$ is more efficient than DPB. \\
\hline Illegal use of middle frame & over 0.886 & over 0.865 & $\mathrm{DH}$ is more efficient than DPB. \\
\hline
\end{tabular}

Table 2. Comparison Value to Identify Content Similarity

\begin{tabular}{|l||c|c|c|}
\hline \multicolumn{1}{|c||}{ Content Format } & $\begin{array}{c}\text { Identification of } \\
\text { Comparison Value }\end{array}$ & DH Comparison Value & DPB Comparison Value \\
\hline \hline \multirow{2}{*}{$\begin{array}{l}\text { Original Content without } \\
\text { Modification }\end{array}$} & Equivalent & $95.1 \% \sim 100 \%$ & $95.1 \% \sim 100 \%$ \\
\cline { 2 - 4 } & Similar & $74.1 \% \sim 95 \%$ & $75.1 \% \sim 95 \%$ \\
\hline \multirow{2}{*}{$\begin{array}{l}\text { Modified Content in Resolution } \\
\text { Modified Content in Format }\end{array}$} & Different & $0 \% \sim 74 \%$ & $0 \% \sim 75 \%$ \\
\cline { 2 - 4 } & Equivalent & $93.1 \% \sim 100 \%$ & $94.1 \% \sim 100 \%$ \\
\hline \multirow{2}{*}{$\begin{array}{l}\text { Content Using A Certain } \\
\text { Frame }\end{array}$} & Similar & $71 \% \sim 93 \%$ & $71 \% \sim 94 \%$ \\
\cline { 2 - 4 } & Different & $0 \% \sim 70 \%$ & $0 \% \sim 70 \%$ \\
\cline { 2 - 4 } & Equivalent & $94.1 \% \sim 100 \%$ & $93.1 \% \sim 100 \%$ \\
\hline
\end{tabular}


Table 3. Performance Comparison in Proposed Method

\begin{tabular}{|l||c|c|c|c|}
\hline \multicolumn{1}{|c|}{ Mechanism } & $\begin{array}{c}\text { Pixel } \\
\text { Value }\end{array}$ & $\begin{array}{c}\text { Luminance } \\
\text { Value }\end{array}$ & $\begin{array}{c}\text { Vector } \\
\text { Value }\end{array}$ & $\begin{array}{c}\text { Proposed } \\
\text { Mechanism }\end{array}$ \\
\hline \hline Comparison Value Extraction from Original Content & 0 & 0 & 0 & 0 \\
\hline $\begin{array}{l}\text { Comparison Value Extraction from the Modified } \\
\text { Content in Format }\end{array}$ & $\Delta$ & 0 & $\Delta$ & 0 \\
\hline $\begin{array}{l}\text { Comparison Value Extraction from the Modified } \\
\text { Content in Resolution }\end{array}$ & 0 & $\Delta$ & $\Delta$ & 0 \\
\hline Comparison Value Extraction from A Certaion Frame & $\Delta$ & 0 & $X$ & 0 \\
\hline Accuracy Judgement of piracy information & 0 & $\Delta$ & $\Delta$ & 0 \\
\hline Comparison Value Extraction of Content Details & $\Delta$ & $\Delta$ & 0 & $\Delta$ \\
\hline
\end{tabular}

$\bigcirc$ : Accuracy, $\triangle$ : Prediction, $\mathrm{X}$ : Flexible Prediction

\section{CONCLUSION}

This paper studied the mechanism to certify the original digital content video for the purpose of protecting the copyright of the digital content copyright holder. The proposed mechanism analogized the modified content of the original content and provided the facts infringed. In other words, the mechanism extracted the same value or similar value out of the items such as theft of original con tent, content color change, content format change, content resolution change, and the theft of any frame (not the representative key frame). We combined pixels with histogram mechanism in order to extract content similarity and sameness. We complemented the shortcomings like pixel pulses and histogram color change so that we could extract content type--specific copyright infringement facts.

As this paper measured the content similarity, the study seems to be of use to protect the copyright of an author if we use this finding as evidence to prove the copyright infringement facts. However, as UCC authors are mostly minors who do not know legal rights or regulations, and currently we do not have legal regulation on whether to determine it as a copyright infringement if the similarity between the original content and the infringed content is over what \%, relevant laws and regulations should be provided in the near future.

\section{REFERENCES}

[1] Tim O'Reilly, "What is Web 2.0 Design Pattern and Business Model for the Next Generation of Software," 2005.

[2] Jing Chae-Duk, Shin Weon, Hong Young-Jin, and Rhee Kyung-Hyune, "Interval Two-dimensional Hash Chains and Application to a DRM system," Journal of Korea Multimedia Society, Vol. 10, No.12, pp. 1663-1670, 2007.

[3] W. Jonker and J.P. Linnarz, "Digital Rights Management in Consuner Electronic Products," Signal Processing Manazine, IEEE, Vol.21, Issue 2, pp. 82-91, 2004.

[4] M. Yeung, B. Yeo, and B. Liu, "Segmentation of Video by Clustering and Graph Analysis," Computer Vision and Image Understanding, Vol.71, No.1, pp. 94-109, 1998.

[5] K. Hang-Bong, "Generation of Video Highlights Using Video Context and Perception," Proc. Of SPIE, Storage and Retrieval for Media Databases 2001, Vol. 4315, pp. 320-399, 2001.

[6] Bovic, and Al C. Handbook of Image Video Processing, Communications, Networking and Multimedia Series, Edition, 2005.

[7] A. Hanjalic and H. Zhang, "An Integrated- 
Scheme for Automated Video Abstraction Based on Unsupervised Cluster-Validity Analysis," IEEE Taans. Cir. \& Sys. for Video Tech, Vol.9, No.8, pp. 1280-1289, 1999.

[8] ISO/IEC 13818-2 MPEG-2, "Generic Coding of Moving Pictures and Associated Audio," 1993.

[9] ISO/IEC 11172-2 MPEG-1, "Information Technology - Coding of Moving Pictures and Associated Audio for Digital Storage Media at up to About 1.5Mbit/s-Video," Geneva, 1993.

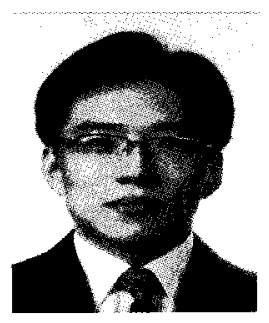

\section{Eun-Gyeom Jang}

Received his Ph.D. degree in Computer Engineering from Daejeon University, Daejeon, Korea in 2007. He is a leader of Technical Laboratory, M2M Korea since 2008. From 2009, he is an adjunct professor at Department of Computer Engineering, Daejeon University. His research interests are DRM, computer forensics, smart grids, and mobile security. 